\section{P22 ADME PROPERTIES OF VAMOROLONE, A FIRST-IN- CLASS DISSOCIATIVE STEROIDAL ANTI-INFLAMMATORY DRUG}

${ }^{1} \mathrm{JM}$ Damsker*, ${ }^{2} Y$ Piyis, ${ }^{2}$ LA Peña, ${ }^{1} \mathrm{JM}$ McCall. ${ }^{1}$ ReveraGen BioPharma Inc., Rockville, MD; ${ }^{2}$ Metis Laboratories, Ronkonkoma, NY, USA

\subsection{6/archdischild-2019-esdppp.60}

Background Vamorolone is a first-in-class dissociative steroidal drug currently in Phase $2 b / 3$ clinical trials in four to sevenyear old boys with Duchenne muscular dystrophy (DMD). Recent published findings from a Phase $2 \mathrm{a}$ study in DMD boys have demonstrated that vamorolone is well-tolerated through the highest dose tested $(6 \mathrm{mg} / \mathrm{kg} /$ day; two-weeks treatment) and shows a similar pharmacokinetic profile to prednisolone. ${ }^{1}$ The objective of the current study was to assess the ADME (absorption, distribution, metabolism, excretion) properties of vamorolone using in vivo quantitative whole-body autoradiography (QWBA) and mass balance experimentation in rats.

Methods For the QWBA study, Long Evans (LE) rats were dosed with ${ }^{14} \mathrm{C}$-labeled vamorolone and sacrificed after a defined time interval ( 6 groups with $n=5$ rats per time interval). Each frozen rat carcass was embedded in a carboxymethylcellulose matrix and cyrosectioned. Autoradiography images were acquired, analyzed, and the radioactivity in each tissue was quantified. For the mass balance study, LE rats $(n=6)$ were dosed with ${ }^{14} \mathrm{C}$-labeled vamorolone. Urine and feces were collected from each animal at defined time intervals.

Results The QWBA study demonstrated a widespread distribution of vamorolone amongst body organs with a peak absorption between 2-6 hours for most structures. In gastrointestinal tract organs, the peak absorption fell between the 6 and 24hour time points. The mass balance study revealed that vamorolone was eliminated to low steady state levels by 5 days post administration in urine and 7 days post administration in feces.

Conclusions This study provides crucial information regarding the ADME properties of vamorolone. The results will help guide the design of a human mass balance study scheduled to take place in late 2019 or early 2020.

\section{REFERENCE}

1. Conklin LS, Damsker JM, Hoffman EP, et al. Phase lla trial in Duchenne muscular dystrophy shows vamorolone is a first-in-class dissociative steroidal anti-inflammatory drug. Pharma Res. 2018; 136: 140-150.

Disclosure(s) Jesse M. Damsker and John M. McCall are employees of ReveraGen BioPharma Inc. and have stock options and founder shares, respectively.

\section{P23 EVALUATION OF EXPOSURE TO VANCOMYCIN IN NEONATES WITH CURRENT DOSING APPROACHES}

${ }^{1} \mathrm{~K}$ Dao*, ${ }^{1,2} \mathrm{M}$ Guidi, ${ }^{1} \mathrm{P}$ Andre, ${ }^{3} \mathrm{E}$ Giannoni, ${ }^{4} \mathrm{~A}$ Fuchs, ${ }^{4} \mathrm{M}$ Pfister, ${ }^{1} \mathrm{~T}$ Buclin, ${ }^{1,2,5} \mathrm{C}$ Csajka.

${ }^{1}$ Clinical Pharmacology, Centre Hospitalier Universitaire Vaudois, Lausanne; ${ }^{2}$ School of Pharmaceutical sciences, University of Geneva, Geneva; ${ }^{3}$ Department Mother-Woman-Child, Centre Hospitalier Universitaire Vaudois, Clinic of Neonatology, Lausanne; ${ }^{4}$ Pediatric Pharmacology and Pharmacometrics, University of Basel Children's Hospital, UKBB, Basel; ${ }^{5}$ Service of Pharmacy, Centre Hospitalier Universitaire Vaudois, Lausanne, Switzerland
Background Several neonatal dosing recommendations for vancomycin are found in the literature, variably based on age, renal function and body weight. Still there is no consensus regarding optimal initial dosing in term or preterm neonates. Our objective was to evaluate and compare how current dosing approaches perform with respect to target plasma concentration attainment, using a comprehensive population PK model of vancomycin developed in a large cohort of neonates.

Methods A single-compartment, linear elimination population pharmacokinetic model incorporating postmenstrual age, kidney function and body weight as covariates was elaborated using NONMEM $^{\circledR}$, based on 1848 vancomycin concentration values measured in 405 neonates during routine TDM. The model was then used to simulate the distribution of vancomycin concentrations resulting from 20 dosing guidelines identified in the literature. Proportions of patients within and above target exposure were used as a performance measure of each dosing regimen, defining target as $\mathrm{AUC}_{24} / \mathrm{MIC}$ ratio of 400 $700 \mathrm{~h}$ and trough concentration of $10-20 \mathrm{mg} / \mathrm{L}$, both on days 1 and 7 of treatment.

Results Only 2 out of 20 current dosing recommendations (Neonatal Formulary 7 and Neofax ${ }^{\circledR}$ meningitis regimens) ensured target attainment in about $60 \%$ of neonates on both days 1 and 7. Most other guidelines produced below-target exposure in a large fraction of patients (22-97\%), except two that frequently produced overexposure (55-66\%).

Conclusion A majority of currently used vancomycin dosing regimens proposed in the literature failed to ensure target attainment in at least $60 \%$ of neonates. It is important that concentration exposure associated with best chances of therapeutic success is promptly reached, in particular in neonatal units having a high prevalence of coagulase negative Staphylococci. We recommend electing an effective dosage regimen, with a loading dose, to ensure early target attainment in a majority of patients. Subsequent therapeutic concentration monitoring remains warranted to further individualize vancomycin dosage and optimize exposure in all patients.

Disclosure(s) Nothing to disclose

\section{P24 CAFFEINE IN PRETERM NEONATES: IMPACT ON SLEEP WAKE REGULATION}

${ }^{1}$ AN Datta* ${ }^{2} \mathrm{G}$ Koch, ${ }^{2} \mathrm{~N}$ Schönfeld, ${ }^{3} \mathrm{~K}$ Jost, ${ }^{2} \mathrm{~A}$ Atkinson, ${ }^{3} \mathrm{SM}$ Schulzke, ${ }^{2} \mathrm{M}$ Pfister. ${ }^{1}$ Pediatric Neurology and Developmental Medicine; ${ }^{2}$ Pediatric Pharmacology and Pharmacometrics Research Center; ${ }^{3}$ Neonatology, University Children's Hospital Basel (UKBB), Basel, Switzerland

\subsection{6/archdischild-2019-esdppp.62}

Background Sleep wake regulation undergoes distinctive maturational changes. Ultradian sleep wake rhythm predominates at preterm age and is mainly driven by the internal clock. Increased perinatal morbidity in preterm neonates often appears with breathing disorders, among which apnea of prematurity (AOP) is the most frequently observed. Pharmacological support with caffeine has been successfully employed in the treatment of AOP. Nowadays, caffeine citrate is administered to all preterm neonates suffering from AOP. Objective of our study was a quantitative investigation of whether caffeine citrate treatment for reduction of apnea and bradycardia of prematurity affects sleep-wake behavior in preterm neonates. 
Methods Observational study of 64 preterm neonates during their first five days of life with gestational age (GA) $<32$ weeks or very low birth weight of $<1500$ g. Total of 52 patients treated with caffeine and 12 controls without caffeine were included. Sleep-wake behavior was scored in wakefulness (W), active sleep (AS), and quiet sleep (QS) associated with physical and cerebral regeneration. Individual caffeine concentration of every neonate was simulated with a pharmacokinetic model.

Results For increasing caffeine concentration, W increased, AS decreased, and QS was unchanged for GA $>$ or $=28$ weeks. No caffeine effect for GA $<28$ weeks could be demonstrated. Maturational effects could be seen when comparing preterm neonates of GA $>32$ weeks with a birth weight of $<1500 \mathrm{~g}$ with very preterm neonates born of GA < 32: Neonates born $>32$ weeks had a significantly higher amount of $\mathrm{W}$ and lower percentage of AS.

Conclusions Treatment of apnea and bradycardia as well as stabilization of respiration with standard caffeine treatment is not at cost of QS, i.e. time for physical and cerebral regeneration during sleep remains unchanged. There is an increased fraction of W, alertness and most probably also arousability.

Disclosure(s) Nothing to disclose

\section{P25 COMPARISON OF RENAL FUNCTION ESTIMATION METHODS IN CRITICALLY ILL CHILDREN: A PILOT STUDY}

${ }^{1} \mathrm{E}$ Dhont, ${ }^{2} \mathrm{~T}$ Van Der Heggen, ${ }^{1} \mathrm{~A}$ De Jaeger, ${ }^{1} \mathrm{~J}$ Willems, ${ }^{3} \mathrm{~S}$ De Baere, ${ }^{3} \mathrm{~S}$ Croubels, ${ }^{4} \mathrm{~J}$ Vande Walle, ${ }^{5} \mathrm{p}$ De Paepe, ${ }^{1,6} \mathrm{p}$ De Cock*. ${ }^{1}$ Paediatric Intensive Care Unit; ${ }^{2}$ Paediatrics Department, Ghent University Hospital; ${ }^{3}$ Faculty of Veterinary Medicine, Department of Pharmacology, Toxicology and Biochemistry, Ghent University; ${ }^{4}$ Paediatric Nephrology Department, Ghent University Hospital; ${ }^{5}$ Heymans Institute of Pharmacology, Ghent University; ${ }^{6}$ Pharmacy, Ghent University Hospital, Ghent, Belgium

\subsection{6/archdischild-2019-esdppp.63}

Background Accurate assessment of renal function is crucial in intensive care to guide therapy. Both acute kidney injury and augmented renal clearance (ARC) may compromise outcome. Common formulas to estimate glomerular filtration rate (GFR) are unreliable in critically ill adults. ${ }^{1}$ A comparison of a gold standard technique to assess GFR with these formula-based estimations has never been reported in pediatric intensive care (PICU) patients. Our aim was to evaluate the feasibility of measuring plasma iohexol clearance $\left(\mathrm{CL}_{\mathrm{IOHEX}}\right)$ for GFR assessment in critically ill children and to compare $\mathrm{CL}_{\text {IOHEX }}$ with estimated GFR using the modified Schwartz formula $\left(\mathrm{eGFR}_{\text {Schwartz }}\right)$.

Methods A prospective, interventional study was conducted at the PICU of the Ghent University Hospital, Belgium. Critically ill children without chronic kidney disease were included. After injection of a weight-dependent bolus of iohexol, serial blood samples $(n=6)$ were taken over a 6-hours interval. $\mathrm{CL}_{\text {IOHEX }}$ was compared to $\mathrm{eGFR}_{\text {Schwartz }}$ Correlation between both methods was assessed by a Pearson's correlation coefficient (r).Bland-Altman plots were evaluated to assess bias and limits of agreement (LOA). ARC was defined as a GFR exceeding normal values for age plus two standard deviations. Results 40 patients, median age 16 months (range 15 days 13,6 years), $72,5 \%$ males, were included. No adverse effects related to iohexol were observed. Median $\mathrm{CL}_{\mathrm{IOHEX}}$ was 121 $\mathrm{ml} / \mathrm{min} / 1.73 \mathrm{~m}^{2}$ (range: $43-221 \mathrm{ml} / \mathrm{min} / 1.73 \mathrm{~m}^{2}$ ). ARC was present in 20 patients based on $\mathrm{CL}_{\text {IOHEX }}$. Median
eGFR $_{\text {Schwartz }}$ was $81 \mathrm{ml} / \mathrm{min} / 1.73 \mathrm{~m}^{2}$ (range: $31-131 \mathrm{ml} / \mathrm{min} /$ $1.73 \mathrm{~m}^{2}$ ). Only 1 patient was identified with ARC by $\mathrm{eGFR}_{\mathrm{Sch}-}$ wartz. eGFR $_{\text {Schwartz }}$ was systematically lower than $\mathrm{CL}_{\mathrm{IOHEX}}$. There was a good correlation between $\mathrm{CL}_{\mathrm{IOHEX}}$ and $\mathrm{eGFR}_{\mathrm{Sch}}$ wartz $(\mathrm{r}=0,69 ; \mathrm{p}<0,01)$. Bias was $34 \mathrm{ml} / \mathrm{min} / 1.73 \mathrm{~m}^{2}$ with LOA $\left(-24,5 ; 93 \mathrm{ml} / \mathrm{min} / 1.73 \mathrm{~m}^{2}\right)$

Conclusion $\mathrm{CL}_{\mathrm{IOHEX}}$ was safely used to measure true GFR in critically ill children. eGFR Schwartz $_{\text {systematically underestimates }}$ GFR, especially in patients with ARC and seems not reliable in this patient population.

\section{REFERENCE}

1. Baptista JP, Neves M, Rodrigues L, Teixeira L, Pinho J, Pimentel J (2014) Accuracy of the estimation of glomerular filtration rate within a population of critically ill patients. J Nephrol. 27:403-410.

Disclosure(s) Nothing to disclose

\section{P26 PILOT ON HARMONISING DOSING RECOMMENDATION FOR TERM AND PRETERM NEONATES IN THE NETHERLANDS (NEODOSE PROJECT)}

${ }^{1} \mathrm{MA}$ de Hoop-Sommen ${ }^{*},{ }^{2} \mathrm{TM}$ van der Zanden, ${ }^{3,4} \mathrm{~K}$ Allegaert, ${ }^{2,5} \mathrm{RB}$ Flint, ${ }^{2}$ SHP Simons, ${ }^{2,5} \mathrm{SN}$ de Wildt, NeoDose Consortium. ${ }^{1} \mathrm{KNMP}$, Den Haag; ${ }^{2}$ Erasmus MC - Sophia Childrens Hospital, Rotterdam, The Netherlands; ${ }^{3} \mathrm{KU}$ Leuven, Leuven, Belgium; ${ }^{4}$ Erasmus MC Sophia Childrens Hospital, Den Haag; ${ }^{5}$ Radboud University Medical Center, Radboud Institute for Health Sciences, Nijmegen, The Netherlands

\subsection{6/archdischild-2019-esdppp.64}

Background Many drugs are used off-label in term and preterm neonates, and dosing recommendations for many drugs are lacking in the Dutch Paediatric Formulary (DPF). This results in widely varying dosing regimens used across neonatal intensive care units (NICUs) in the Netherlands. The Neodose pilot project aimed to develop best-evidence national dosing recommendations for (pre)term neonates. Because scientific evidence is scarce, a consensus-based approach was used.

Methods A priority drug list, containing the most frequently used drugs for neonates, was drafted. From this list 22 drugs were selected for further research within the Neodose pilot project. The pilot utilized a two-step approach: First, consensus was established with all Dutch NICUs for neonatal dosing recommendations. Local treatment protocols were retrieved, compared and discussed, leading to consensus-based dosing recommendations. Secondly, we aimed to develop best-evidence dosing recommendations for the following five drugs: acyclovir, ganciclovir, ibuprofen, hydrocortisone and dexamethasone.

Results For 21 of 22 drugs, local dosing guidelines differed significantly. Mostly concerning total daily dose, dosing frequency and route of administration. Little or no distinction is made between treatment of preterm and term neonates. Approximately half of the consensus-based dosing recommendations (45\%) differ in some degree from all local protocols. Comparing the consensus-based dosing recommendations with the available evidence, almost half of the consensus doses were adjusted. The grounds on which dosing recommendations were adjusted differed. Acyclovir-dosing adjustment was based on pharmacokinetics. Hydrocortisone-dosing was adjusted due to new insights after the evidence has been put together. For dexamethasone-dosing, the consensus dose was eventually chosen, because every available trial used a different dosing regimen. 